\section{Precentral Gyrus}

John Bigbee

Anatomy and Neurobiology, Virginia

Commonwealth University, Richmond, VA, USA

\section{Synonyms}

Motor strip; Primary motor area

\section{Definition}

Located in the frontal lobe of the cerebral cortex, Frontal Lobes

the precentral gyrus is a vertically oriented, cere- $>$ Homunculus

bral convolution located immediately anterior to, and running parallel with, the central sulcus. The precentral gyrus is the primary somatomotor motor cortex and sends efferent axons in the corticospinal tract to terminate on cranial and spinal motor neurons. The body is somatotopically represented on the gyrus in an inverted pattern with regions controlling the head located in the lower portions. Lesions in the precentral gyrus may result in weakness or paralysis in the corresponding region of the body.

\section{Cross-References}

